\title{
Low pressure cardiac tamponade: diagnosis facilitated by Doppler echocardiography
}

\author{
Sharonne N Hayes, William K Freeman, Bernard J Gersh
}

\begin{abstract}
A case of low pressure cardiac tamponade is reported in which hypovolaemia altered the clinical findings of tamponade and complicated the diagnosis. Venous pressure was not raised and there was no pulsus paradoxus. In this patient, a man of 67 , characteristic variations in diastolic inflow signals and the isovolumic relaxation period throughout the respiratory cycle were shown by Doppler echocardiography and led to the diagnosis of cardiac tamponade in the absence of characteristic signs and symptoms.
\end{abstract}

Cardiac tamponade is defined as cardiac compression caused by an accumulation of pericardial fluid that evokes and ultimately defeats compensatory mechanisms, resulting in haemodynamic compromise. Although the signs and symptoms of cardiac tamponade are relatively non-specific, the combination of dyspnoea, tachycardia, and hypotension with an almost universally raised central venous pressure and pulsus paradoxus usually leads the clinician strongly to suspect the diagnosis We describe a patient in whom the signs and symptoms of tamponade were masked by hypovalaemia.

\section{Case report}

A 67 year old white man was admitted to the hospital after an episode of syncope with persistent hypotension. Thirteen days before admission, urgent coronary artery bypass surgery was performed for unstable angina. At that time, angiography showed moderate left ventricular dysfunction with an ejection fraction of $32 \%$ and severe occlusive disease of the right and left anterior descending coronary arteries. Saphenous vein grafting of the right coronary artery and left internal mammary grafting to the left anterior descending was uncomplicated. The patient was discharged five days before the current admission after an uneventful recovery. Discharge medications included aspirin $80 \mathrm{mg}$ and a diuretic formulation of hydrochlorothiazide $25 \mathrm{mg}$ and triamterene $50 \mathrm{mg}$, once daily.

The patient did not have any chest discomfort after operation. On the morning of admission, he experienced progressive dyspnoea and nausea, followed by an episode of syncope.

Evaluation in the local emergency room showed the patient to be tachycardic with a systolic blood pressure of $70 \mathrm{~mm} \mathrm{Hg}$ Intravenous dopamine $(6 \mu \mathrm{g} / \mathrm{kg} / \mathrm{min}$ infusion) and infusion of physiological saline transiently increased the systolic blood pressure to $90 \mathrm{~mm} \mathrm{Hg}$.

Upon transfer to our hospital, the patient was alert and complained of dyspnoea. Blood pressure was $80 / 60 \mathrm{~mm} \mathrm{Hg}$ without pulsus paradoxus. The heart rate was regular at 110 beats/minute and the respiratory rate was 18 / minute. The mucous membranes were dry, there was generalised pallor, and poor skin turgor. The lungs were clear and the sternotomy well healed. There was no jugular venous distension or Kussmaul's sign. The cardiac examination showed a grade $2 / 6$ apical systolic murmur that radiated to the axilla suggestive of mild mitral regurgitation and a $2 / 6$ systolic ejection murmur at the left lower sternal border. There was no rub, gallop, or extra sound.

The electrocardiogram showed sinus tachycardia, an old inferior $Q$ wave infarction, and $T$ wave inversion in leads $V 2-V 6$; all unchanged from the earlier postoperative tracings. The chest $x$ ray showed moderate cardiomegaly and clear lung fields. The haemoglobin concentration and serum concentrations of creatine kinase and lactate dehydrogenase were within normal limits.

Intravenous infusion of one litre of physiological saline had no effect on the pulse or blood pressure; attempts to taper the

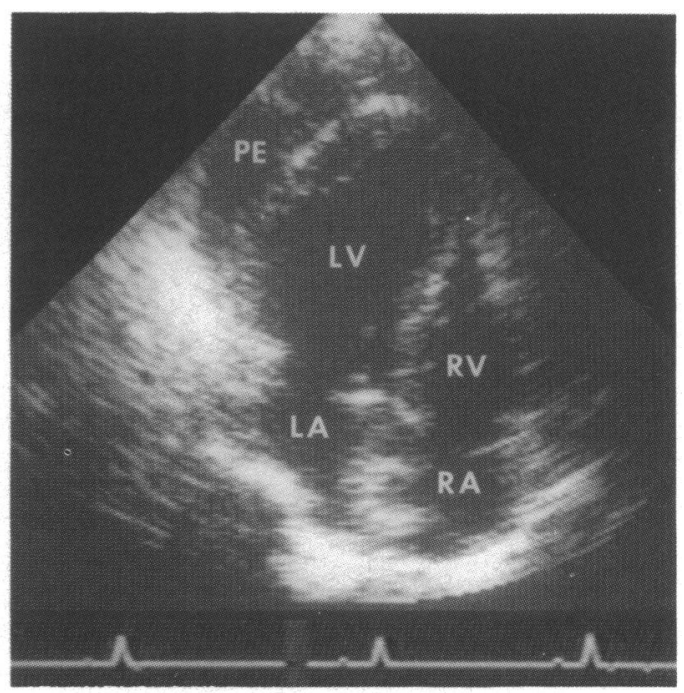

Figure 1 Four chamber cross sectional echocardiographic image of a moderate localised inferolateral apical pericardial effusion $(P E)$. No diastolic compression of the free walls of the right ventricle $(R V)$ or right atrium $(R A)$ was evident. $L V$, left ventricle, $L A$, left atrium. 


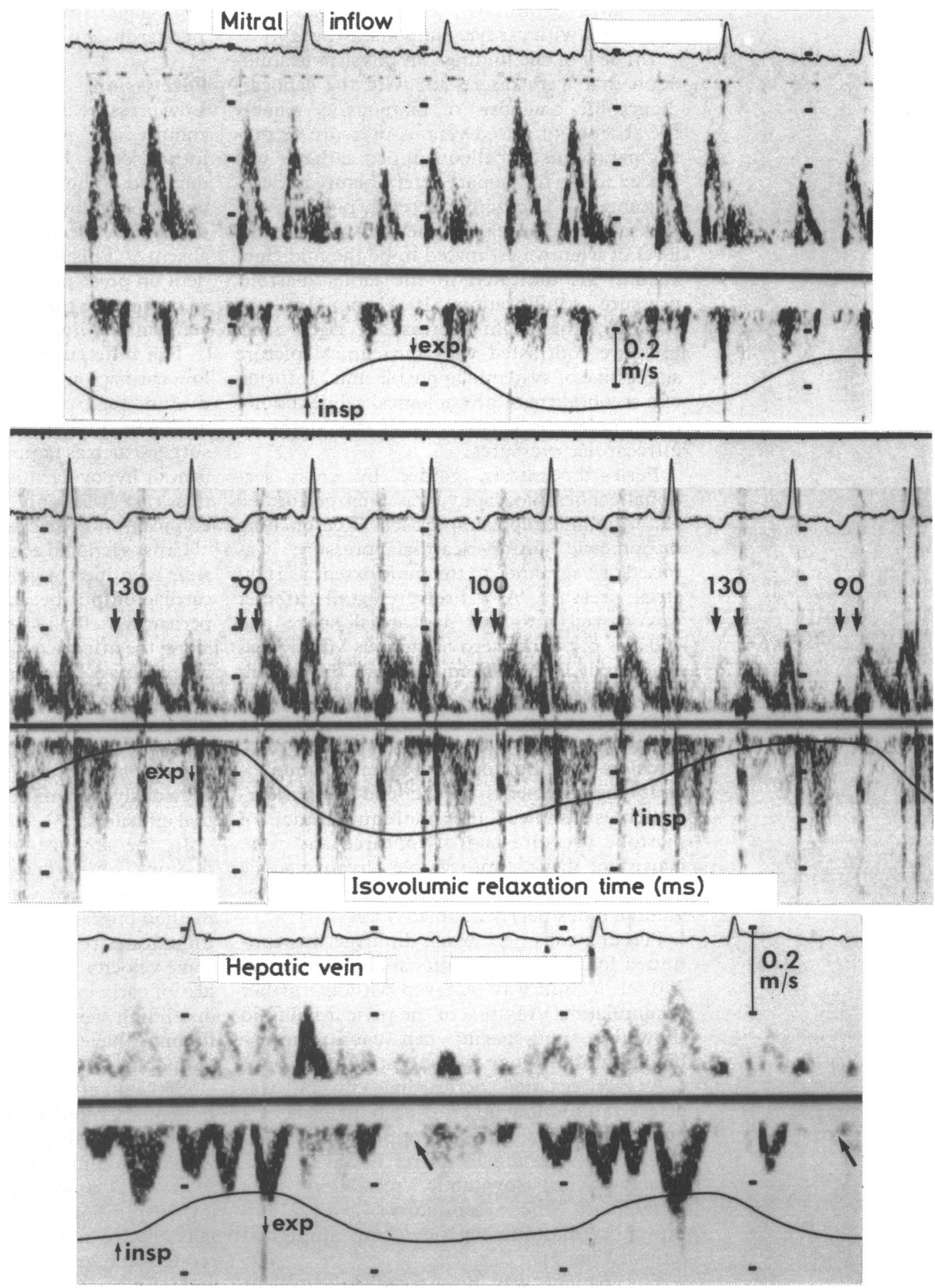

Figure 2 Pulsed wave Doppler findings before pericardiocentesis. Respirometry tracing indicates the onset of inspiration (insp) and expiration (exp). Early mitral inflow velocity fell with inspiration and increased considerably during the first beat after expiration. There was significant increase in isovolumic relaxation time (between pairs of arrows) with inspiration, with shortening occurring during the first beat after expiration. There was no diastolic hepatic venous filling (arrows) during the first beat after expiration.

dopamine infusion led to progressive hypotension.

Cross sectional echocardiography showed mild left ventricular enlargement with moderately decreased function and an estimated ejection fraction of $30-35 \%$ (similar to the preoperative study). The inferior septum was akinetic, with severe hypokinesis of the inferolateral segments from base to apex and moderate hypokinesis of the anteroseptal and anterior segments. There was a moderate localised inferolateral apical pericardial effusion with strands evident (fig 1). There was no diastolic compression of the right ventricular or atrial free walls.

Pulsed wave Doppler examination with respirometry showed a decrease of nearly $50 \%$ in mitral $\mathrm{E}$ wave velocity during early inspiration with accompanying prolongation of the isovolumic relaxation time. The velocity of the mitral $E$ wave increased sharply with the first beat after expiration and the isovolumic relaxation time shortened. There was no hepatic venous diastolic filling during the first 
beat after expiration. These findings were consistent with cardiac tamponade (fig 2).

In view of the findings on physical examination that were discrepant with the echocardiographic diagnosis of tamponade, namely the absence of raised venous pressure or pulsus paradoxus, a balloon tipped catheter was placed in the pulmonary artery before pericardiocentesis. The zero reference point of the pressure transducer was the mid-chest, at the level of what we estimated to be the mid right atrium. As indicated in the table, diastolic pressure equilibration $(10-11 \mathrm{~mm} \mathrm{Hg})$ was present. The slight increase in right atrial pressure contrasted with the clinical picture suggestive of systemic hypovolaemia. Infusion of a second litre of physiological saline did not result in an appreciable change in systemic or intracardiac pressures.

Pericardiocentesis, guided by cross sectional echocardiography, was then performed via a modified apical approach. The opening manometric intrapericardial pressure was raised $(12 \mathrm{~cm}$ fluid) to the same extent as right atrial pressure. A 7 French pigtail catheter was passed into the pericardial space and $450 \mathrm{ml}$ of dark serosanguinous fluid was evacuated. During fluid removal, the patient immediately noted a considerable improvement in his dyspnoea, the systemic blood pressure increased, and dopamine infusion could be stopped. Repeat haemodynamic measurements at the end of pericardiocentesis showed that the equilibration of diastolic pressure had disappeared and were consistent with appreciable hypovolaemia (right atrial pressure of $0 \mathrm{~mm} \mathrm{Hg}$ ); the strokevolume index nearly doubled (table).

Percutaneous pericardial drainage was continued for another 48 hours and an additional $450 \mathrm{ml}$ of fluid was removed without further accumulation. Analysis of the pericardial fluid showed a non-specific cell count; microbiology and cytology were negative.

A tiny residual pericardial effusion was detected on follow up cross sectional echocardiography. Pulsed wave Doppler showed no significant variation in mitral $\mathrm{E}$ wave inflow velocities or isovolumic relaxation time throughout the respiratory cycle, and expiratory diastolic hepatic venous filling had returned (fig 3).

The patient was discharged in excellent condition four days after admission. The patient's cardiopulmonary state was stable at four months follow up. Cross sectional echocardiography at that time showed no recurrent pericardial effusion; the results of pulsed wave Doppler with respirometry were unchanged from the study immediately after pericardiocentesis.

\section{Discussion}

Low pressure cardiac tamponade is caused by a compressive pericardial effusion when the intravascular blood volume is considerably depleted. ${ }^{1}$ The central venous pressure is normal or often low, and hence the jugular venous distension, seen in classic tamponade, is absent. ${ }^{12}$ Pulsus paradoxus, although dependent on pulse pressure, is another clinical sign of tamponade that is often absent when there is concomitant hypovolaemia. ${ }^{12}$

The differential diagnosis of this patient's low cardiac output state included myocardial dysfunction from infarction or ischaemia and a mechanical complication of coronary bypass surgery such as tamponade, pulmonary embolism, or hypovolaemia. Physical examination in this case was only partly informative, and atypical for cardiac tamponade.

Cross sectional echocardiography at the bedside identified a potential cause of the low cardiac output because it showed a moderate pericardial effusion with primary localisation along the inferolateral left ventricle. There was no excessive "swinging" motion of the heart or diastolic compression of the free wall of the right chamber. In this patient, the cross sectional echocardiogram was not at all diagnostic of tamponade; an inconsistency which has been previously recognised in postoperative pericardial effusions. ${ }^{3}$

In the absence of typical cross sectional findings of cardiac tamponade, ${ }^{4-6}$ Doppler echocardiography was the crucial non-invasive method of reaching a diagnosis in this patient. Significant respiratory variation in mitral $\mathrm{E}$ wave velocity, isovolumic relaxation time, and loss of early expiratory diastolic systemic venous filling were detected by Doppler. These findings have been associated with cardiac tamponade. $^{78}$

Appleton et al correlated the dissociation of pulmonary capillary wedge filling pressure and intrapericardial pressure during respiration with mitral inflow velocities measured by pulsed wave Doppler in cardiac tamponade. ${ }^{7}$ During inspiration, pulmonary wedge pressure falls relative to intrapericardial pressure and so reduces the gradient between the left atrium and left ventricle in diastole. Because Doppler flow velocities are directly related to the driving pressure gradient, the early mitral $\mathrm{E}$ wave velocity also falls appreciably with the onset of inspiration. The isovolumic relaxation time also increases with inspiration, reflecting delay of mitral valve opening with the decrease in

Results of catheter studies

\begin{tabular}{llc}
\hline & Before pericardiocentesis & After pericardiocentesis \\
\hline Heart rate (beats/min) & 110 & 70 \\
Pressure $(\mathrm{mm} \mathrm{Hg}):$ & $70 / 50$ & $100 / 60$ \\
Systemic arterial & 10 & 0 \\
Mean right atrial & $26 / 11$ & $20 / 1$ \\
Right ventricle & $25 / 11$ & $20 / 2$ \\
Pulmonary artery & 10 & 5 \\
Pulmonary capillary wedge & $2 \cdot 6$ & $3 \cdot 2$ \\
Cardiac index $\left(1 /\right.$ min $\left./ \mathrm{m}^{2}\right)$ & 24 & 46 \\
Stroke volume index $\left(\mathrm{ml} /\right.$ beat $\left./ \mathrm{m}^{2}\right)$ & 12 & 1 \\
Mean intrapericardial pressure $\left(\mathrm{cm} \mathrm{H}_{2} \mathrm{O}\right)$ & & \\
\hline
\end{tabular}



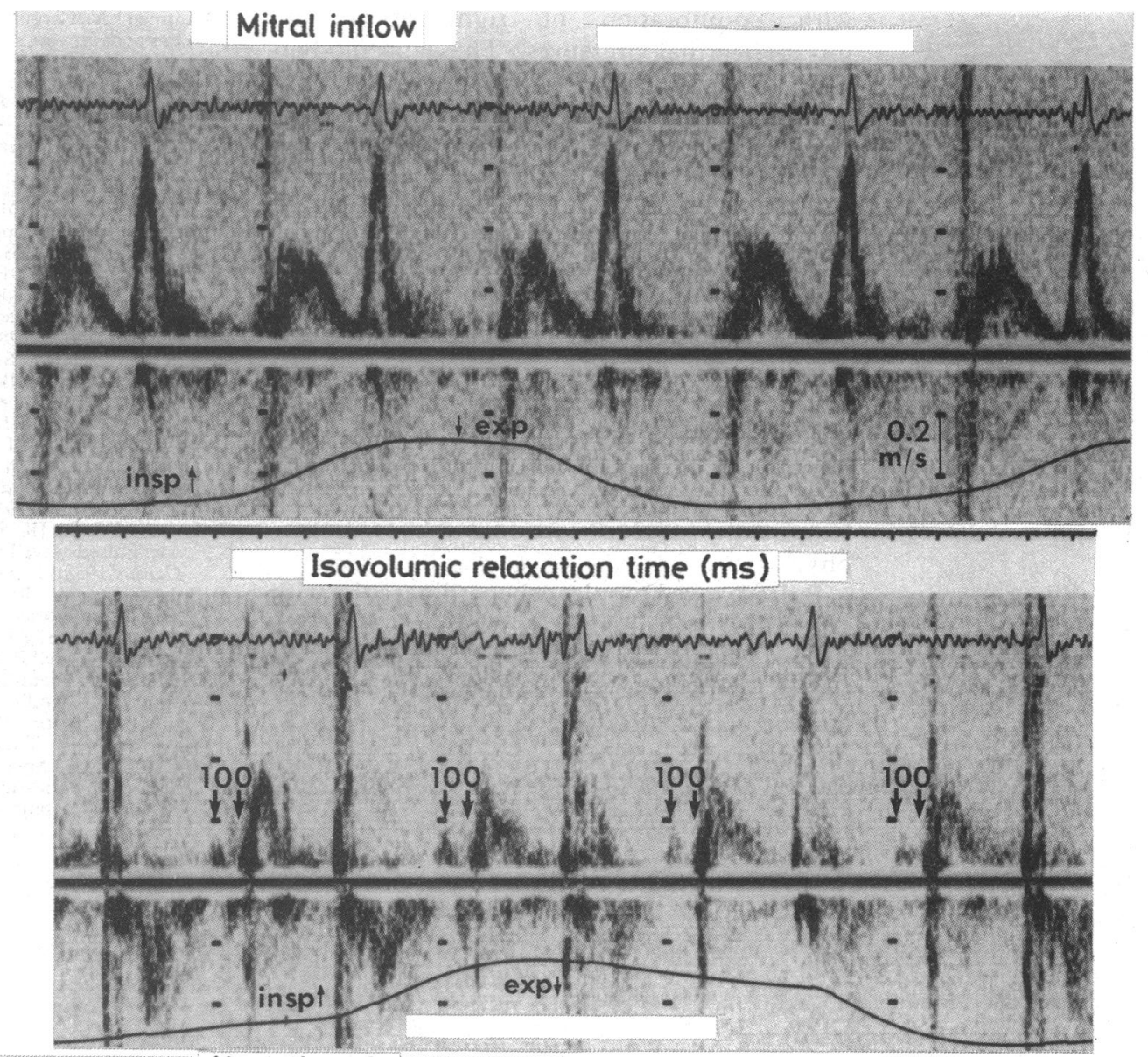

\section{Hepatic vein}

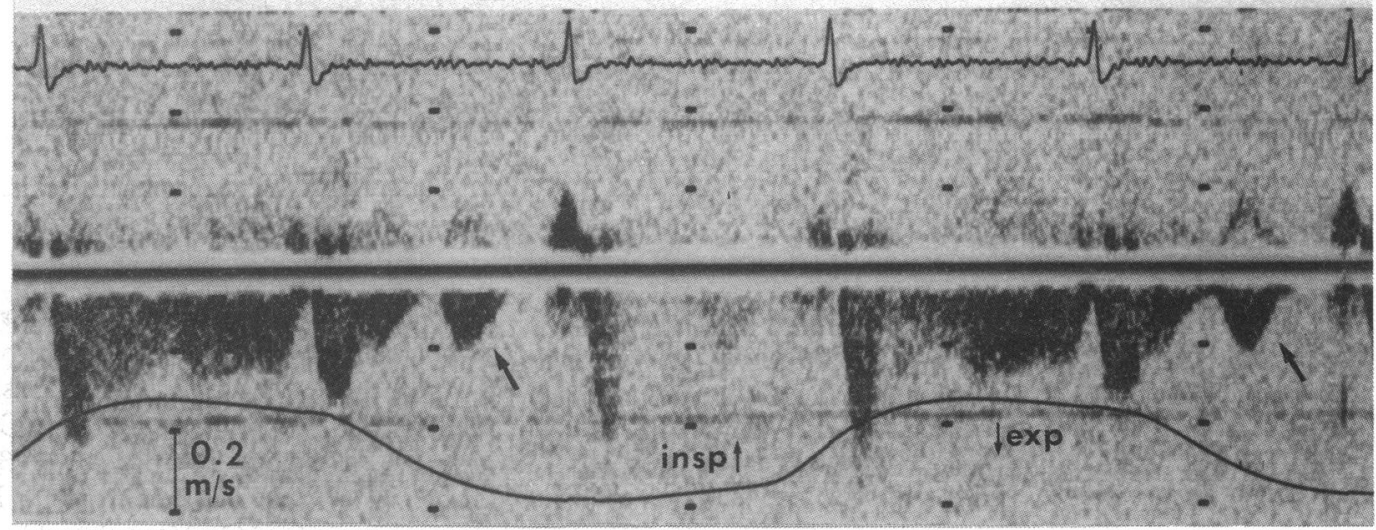

Figure 3 Pulsed wave Doppler findings after pericardiocentesis. Respirometry tracing shows the onset of inspiration (insp) and expiration (exp). There was no appreciable respiratory variation in early mitral inflow velocities. The isovolumic relaxation time (between pairs of arrows) was constant throughout the respiratory cycle. Diastolic hepatic venous filling (arrows) in early expiration was restored.

pulmonary venous filling pressure. In addition, these changes reciprocally interrelate with changes in the right heart where early diastolic filling velocities are increased across the tricuspid valve with inspiration and decreased with expiration. ${ }^{7}$ This reciprocal ventricular interdependence is probably the result of respiratory augmentation of venous return in the face of restricted intracardiac volume changes caused by extracardiac compression. Neither the mitral $\mathrm{E}$ wave velocity nor the isovolumic relaxation time vary significantly with respiration in patients without tamponade. ${ }^{7}$

Early expiratory decrease or loss of systemic venous diastolic inflow velocities by Doppler has also been detected in tamponade. ${ }^{7}$ This finding correlates with the nadir of the tricuspid early inflow velocity, presumably due to maximisation of impedance to right ventricular filling during early expiration.

In our laboratory the same findings were seen in patients with tamponade studied with pulsed wave Doppler and respirometry. ${ }^{8}$ These Doppler findings consistently resolve after pericardiocentesis. ${ }^{78}$

The diagnosis of "low pressure cardiac tamponade" was confirmed by invasive haemodynamic measurements in this patient. The expanding intrapericardial volume in the setting of hypovolaemia precipitated cardiac tamponade with the diastolic pressure equilibration at low intracardiac pressures and 
with equilibration of right atrial and intrapericardial pressures. These haemodynamic findings have been described before. ${ }^{191}$

It has been reported that low pressure tamponade, despite occurring in hypovolumic states, does not respond to volume repletion. ${ }^{29}$ This was seen in this patient, presumably because of the development of a steep intrapericardial pressure-volume curve, curtailing cardiac filling volume. ${ }^{9}$

Pericardial effusions are common after cardiac surgery but they rarely cause clinical cardiac tamponade. ${ }^{11}$ Haemodynamically significant postoperative pericardial effusions, however, can be loculated, small in volume, and often fail to present the usual signs of tamponade on cross sectional echocardiography.

Pulsed wave Doppler echocardiography with respirometry can greatly facilitate the noninvasive diagnosis of cardiac tamponade. As shown by this case, Doppler echocardiography may be particularly useful for the diagnosis of low pressure cardiac tamponade when the clinical examination and even cross sectional echocardiography may be misleading.
1 Antman EM, Cargill V, Grossman W. Low-pressure cardiac tamponade. Ann Intern Med 1979;91:403-6.

2 Boltwood CM, Lee PY, Tei C, Shah PM. Low-pressure cardiac tamponade. N Engl J Med 1983;309:667-8.

3 D'Cruz IA, Kensey K, Campbell C, Replogle R, Jain M. Two-dimensional echocardiography in cardiac tamponade occurring after cardiac surgery. $\mathrm{J} \mathrm{Am} \mathrm{Coll} \mathrm{Cardiol}$ 1985;5:1250-2.

4 Armstrong WF, Schilt BF, Helper DJ, Dillon JC, Feigenbaum $\mathrm{H}$. Diastolic collapse of the right ventricle with cardiac tamponade: an echocardiographic study. Circulation 1982;65:1491-6.

5 Gillam LD, Guyer DE, Gibson TC, King ME, Marshall JE Weyman AE. Hydrodynamic compression of the right Weyman AE. Hydrodynamic compression of the right atrium: a new echocardiographic

6 Singh S, Wann LS, Schuchard GH, et al. Right ventricular and right atrial collapse in patients with cardiac tamponade-a combined echocardiographic and hemodynamic study. Circulation 1984;70:966-71.

7 Appleton CP, Hatle LK, Popp RL. Cardiac tamponade and pericardial effusion: respiratory variation in transvalvular flow velocities studied by Doppler echocardiography. $J$ Am Coll Cardiol 1988;11:1020-30.

8 Burstow DJ, Oh JK, Seward JB, Tajik AJ. Cardiac tamponade: pulsed-wave Doppler findings [Abstract]. J Am Coll Cardiol 1988;11:75A

9 Boltwood CM, Ryan M. Volume expansion versus pericardiocentesis in low-pressure cardiac tamponade. Am J Med 1987;83:1007-8.

10 Levine MJ, Lorell BH, Diver DJ, Come PC. Low-pressure tamponade identified by echocardiography: hemodynamic results and outcome after pericardiocentesis [Absamic results and outcome after pericardiocente

11 Weit]. Circulation 188,78 Glassman E, Spencer FC. The incidence and natural history of pericardial effusion after cardiac surgery-an echocardiographic study. Circulation 1984;69:506-11.

NOTICES

\section{British Cardiac Society}

The Annual General Meeting will take place at the English Riviera Centre, Torquay on 22 to 25 May 1990. The closing date for receipt of abstracts was 19 January 1990.

\section{Cardiac Doppler}

The Fifth International Congress of the International Cardiac Doppler Society will take place in Trondheim on 13 to 15 September 1990. Inquiries to Congress Secretariat, The Norwegian Institute of Technology, Department of Continuing Education, N-7034 Trondheim, Norway.

\section{Cardiology and cardiac surgery}

A workshop on Update in Cardiology and Cardiac Surgery: East-West Meet will be held at the Institute of Cardio-Vascular Diseases, Madras on 11 to 13 January 1991. Further information from $\mathrm{Dr} \mathrm{K} M$ Cherian, Institute of Cardio-Vascular Diseases, 180 NSK Salai, Madras - 26, India. 